4. E. Kamke, Differentialgleichungen, Lösungsmethoden und Lösungen, 3rd ed., Chelsea, New York, 1948.

5. P. Lévy, Sur la convergence absolue des séries de Fourier, Compositio Math. 1 (1934), 1-14.

6. G. Szegö, Orthogonal polynomials, Amer. Math. Soc. Colloq. Publ. Vol. 23, Amer. Math. Soc., Providence, R. I., 1939.

7. G. N. Watson, A treatise on the theory of Bessel functions, 2nd ed., Cambridge Univ. Press, Cambridge, 1944.

8. N. Wiener, Tauberian theorems, Ann. of Math. (2) 33 (1932), 1-100.

UNIVERSITY OF Michigan

\title{
A COMPARISON THEOREM FOR SPACES OF ENTIRE FUNCTIONS
}

\section{LOUIS DE BRANGES}

A Hilbert space, whose elements are entire functions, is of particular interest if it has these properties:

(H1) Whenever $F(z)$ is in the space and has a nonreal zero $w$, the function $F(z)(z-\bar{w}) /(z-w)$ is in the space and has the same norm as $F(z)$.

(H2) For every nonreal number $w$, the linear functional defined on the space by $F(z) \rightarrow F(w)$ is continuous.

(H3) Whenever $F(z)$ is in the space, the function $F^{*}(z)=\bar{F}(\bar{z})$ is in the space and has the same norm as $F(z)$. If $E(z)$ is an entire function which satisfies the inequality

$$
|E(\bar{z})|<|E(z)|
$$

for $y>0(z=x+i y)$, let $E(z)=A(z)-i B(z)$, where $A(z)$ and $B(z)$ are entire functions which are real for real $z$, and

$$
K(w, z)=[B(z) \bar{A}(w)-A(z) \bar{B}(w)] /[\pi(z-\bar{w})] .
$$

Let $\mathcal{H}(E)$ be the corresponding set of entire functions $F(z)$ such that

$$
\|F\|^{2}=\int|F(t) / E(t)|^{2} d t<\infty
$$

with integration on the real axis, and

$$
|F(z)|^{2} \leqq\|F\|^{2} K(z, z)
$$

for all complex $z$. Then, $\mathfrak{F}(E)$ is a Hilbert space of entire functions

Received by the editors June 29, 1961 and, in revised form, April 5, 1962. 
which satisfies (H1), (H2), and (H3). For every complex number $w$, $K(w, z)$ belongs to $\mathfrak{H C}(E)$ as a function of $z$, and

$$
F(w)=\langle F(t), K(w, t)\rangle
$$

holds for every $F(z)$ in $\mathscr{C}(E)$. As shown in [2], a Hilbert space, whose elements are entire functions, which satisfies (H1), (H2), and (H3), and which contains a nonzero element, is equal isometrically to $\mathfrak{H C}(E)$ for some such choice of entire function $E(z)$ which satisfies (1).

Let

$$
m(t)=\left(\begin{array}{ll}
\alpha(t) & \beta(t) \\
\beta(t) & \gamma(t)
\end{array}\right)
$$

be a given nondecreasing, matrix valued function, defined for $0<t \leqq 1$, with absolutely continuous, real valued entries, such that $\alpha(t)>0$ for $t>0$ and $0=\alpha(0)=\lim \alpha(t)$ as $t \searrow 0$. A real number $b$ in $(0,1)$ is said to be singular with respect to $m(t)$ if it belongs to an open interval $(a, c)$ such that

$$
[\beta(c)-\beta(a)]^{2}=[\alpha(c)-\alpha(a)][\gamma(c)-\gamma(a)] .
$$

Otherwise, a number $b$ in $(0,1]$ is said to be regular with respect to $m(t)$. We write

$$
I=\left(\begin{array}{rr}
0 & -1 \\
1 & 0
\end{array}\right)
$$

Let $E(z)$ be a given entire function which satisfies (1), has no real zeros, and has value 1 at the origin. As shown in $[5 ; 6]$, it determines a family $(E(t, z))$ of entire functions which satisfy $(1), 0<t \leqq 1$, and which have these properties: each function $E(a, z)$ has no real zeros and has value 1 at the origin; for each complex number $w, E(t, w)$ is a continuous function of $t$; when $0<a<b \leqq 1$,

$$
(A(b, w), B(b, w)) I-(A(a, w), B(a, w)) I
$$

$$
=w \int_{a}^{b}(A(t, w), B(t, w)) d m(t)
$$

holds for some choice of matrix valued function $m(t)$, as above; $\lim K(a, w, w)=0$ as $a \searrow 0 ; E(1, z)=E(z)$ identically. When $a<b$ are regular points with respect to $m(t), \mathfrak{H}(E(a))$ is contained isometrically in $\mathfrak{H}(E(b))$. The family $(E(t, z))$ so constructed is uniquely determined by $E(z)$ except for the choice of parametrization.

If $E(z)$ is an entire function which satisfies (1), has no real zeros, and has value 1 at the origin, let $\phi(x)$ be the continuous determination 
of the phase of $\bar{E}(x)$ which vanishes at the origin. The phase function is a strictly increasing function of $x$ which determines $E(z)$ except for a factor of an entire function real for real $z$ and without zeros. Therefore, a knowledge of $\phi(x)$ is sufficient in principle to determine $m(t)$. In applications discussed in [10], it is important to evaluate the number $\tau$ associated with $\phi(x)$ by

$$
\tau=\int_{0}^{1}\left[\alpha^{\prime}(t) \gamma^{\prime}(t)-\beta^{\prime}(t)^{2}\right]^{1 / 2} d t
$$

when it is finite. Because of Theorem $X$ of [4] and connections between growth and distribution of zeros for entire functions of exponential type, Boas [1, Chapter 8], the formula $\tau=\lim \phi(x) / x$ is valid, as $|x| \rightarrow \infty$, in some, but not all, cases in which the limit exists. Estimates of $\tau$ might be made if a valid converse were found for the following interpretation of the classical Sturm-Liouville comparison theorem. See [6] for the relationship of Hilbert spaces of entire functions to second-order differential equations.

THEOREM I. Let $E(z)$ be a given entire function which satisfies (1), has no real zeros, and has value 1 at the origin, and let $(E(t, z))$ be the corresponding entire functions satisfying (2). For each fixed $t, 0<t \leqq 1$, let $\phi(t, x)$ be the phase function associated with $E(t, z)$. Then for each fixed $x, \phi(t, x)$ is a continuous function of $t$ such that

$$
\begin{aligned}
\phi(b, x)-\phi(a, x)= & x \int_{a}^{b} \cos ^{2} \phi(t, x) d \alpha(t) \\
& +2 x \int_{a}^{b} \cos \phi(t, x) \sin \phi(t, x) d \beta(t) \\
& +x \int_{a}^{b} \sin ^{2} \phi(t, x) d \gamma(t),
\end{aligned}
$$

whenever $0<a<b \leqq 1$, and $\lim \phi(t, x)=0$ as $t \searrow 0$.

TheOREM II. For each $i=1,2$, let $E_{i}(z), \phi_{i}(x), m_{i}(t)$, etc., be as in Theorem I. If the matrix inequality

$$
m_{1}^{\prime}(t) \leqq m_{2}^{\prime}(t)
$$

holds almost everywhere in $(0,1)$, then

$$
\phi_{1}(x) / x \leqq \phi_{2}(x) / x
$$

holds for all real $x$.

An unsolved problem (which it is the main purpose of this paper 
to state) is to determine whether some inequality like (5) on phase functions can be used to obtain (4), which implies that $\tau_{1} \leqq \tau_{2}$.

Proof of Theorem I. From (2) we find that $A(t, x)$ and $B(t, x)$ are absolutely continuous functions of $t$ for each fixed $x$ and

$$
\begin{aligned}
B^{\prime}(t, x) & =x A(t, x) \alpha^{\prime}(t)+x B(t, x) \beta^{\prime}(t) \\
-A^{\prime}(t, x) & =x A(t, x) \beta^{\prime}(t)+x B(t, x) \gamma^{\prime}(t)
\end{aligned}
$$

holds almost everywhere. Since

$$
\tan \phi(t, x)=B(t, x) / A(l, x),
$$

$\phi(t, x)$ is an absolutely continuous function of $t$ for each fixed $x$ and

$$
\begin{aligned}
\phi^{\prime}(t, x)= & x \cos ^{2} \phi(t, x) \alpha^{\prime}(t) \\
& +2 x \cos \phi(t, x) \sin \phi(t, x) \beta^{\prime}(t) \\
& +x \sin ^{2} \phi(t, x) \gamma^{\prime}(t)
\end{aligned}
$$

almost everywhere. This implies (3). It follows that $\phi(a, x) / x$ is a nondecreasing function of $a$ for each fixed $x$. Therefore, $\phi(0, x)$ $=\lim \phi(a, x)$ exists as $a \searrow 0$. But $-i B(a, z) / A(a, z)$ is analytic for $y>0$ and has a non-negative real part because of (1). By the Poisson representation, there is a number $p(a) \geqq 0$ such that

$$
\begin{aligned}
& {[B(a, z) / A(a, z)-\bar{B}(a, w) / A(a, w)] /(z-\bar{w})} \\
& \quad=p(a)-\sum(t-z)^{-1}\left(t-\bar{w}^{-1} B(a, t) / A^{\prime}(a, t),\right.
\end{aligned}
$$

where the derivative of $A(a, z)$ is taken with respect to $z$ and summation is over the zeros $t$ of $A(a, z)$. When $z$ and $w$ are real, this formula may be written in the form

$$
[\tan \phi(a, z)-\tan \phi(a, w)] /(z-w)=p(a)+\sum(t, z)^{-1}(t, w)^{-1} \phi^{\prime}(a, t)^{-1},
$$

where the derivative of $\phi(a, t)$ is taken with respect to $t$ and summation is over values of $t$ such that $\phi(a, t) \equiv \pi / 2$ modulo $\pi$. By monotonic properties of the convergence, the same formula must hold in the limit $a=0$. Since $\alpha(a)=p(a)+\sum t^{-2} \phi^{\prime}(a, t)^{-1}$ goes to zero with $a$ by hypothesis, this formula implies that $\phi(0, x)=0$ identically.

Proof of Theorem II. The conclusion of the theorem will first be derived in a simple special case. More general cases can then be obtained by approximation.

Suppose that each $m_{i}(t)$ is piecewise linear and that the non-negative matrix

$$
\left[m_{2}(b)-m_{2}(a)\right]-\left[m_{1}(b)-m_{1}(a)\right]
$$

is strictly positive (in the sense of having kernel zero) whenever 
$0 \leqq a<b \leqq 1$. If $(a, b)$ is a common interval of linearity for $m_{1}(t)$ and $m_{2}(t)$, and if we can always deduce that $\phi_{1}(b, x) / x \leqq \phi_{2}(b, x) / x$ from the hypothesis that $\phi_{1}(a, x) / x \leqq \phi_{2}(a, x) / x$, then (5) can be obtained by recursion in a finite number of steps from the trivial inequality $\phi_{1}(0, x) / x \leqq \phi_{2}(0, x) / x$. To see that this conclusion is indeed valid, observe that $\phi_{i}(t, x)$ is a continuously differentiable function of $t$ in $(a, b)$, for each fixed $x$, and that

$$
\begin{aligned}
\phi_{i}^{\prime}(t, x) / x= & \alpha_{i}^{\prime} \cos ^{2} \phi_{i}(t, x) \\
& +2 \beta_{i}^{\prime} \cos \phi_{i}(t, x) \sin \phi_{i}(t, x) \\
& +\gamma_{i}^{\prime} \sin ^{2} \phi_{i}(t, x) .
\end{aligned}
$$

Therefore, $\phi_{2}(t, x) / x-\phi_{1}(t, x) / x$ is a continuously differentiable function of $t$ whose derivative is strictly positive at all zeros. If this difference is non-negative for $t=a$, it must certainly remain so for $t=b$. This proves the theorem when each $m_{i}(t)$ is piecewise linear and (6) is strictly positive. The case in which (6) is not strictly positive follows by continuity.

Now consider a special case in which each $\gamma_{i}(0)=\lim \gamma_{i}(t)$ is finite as $t \searrow 0$. Because $\alpha_{i}(0)$ is finite and $m_{i}(t)$ is nondecreasing, this condition implies the existence of $\beta_{i}(0)=\lim \beta_{i}(t)$. For each $n=1,2,3, \cdots$, let $m_{\text {in }}(t)$ be the matrix valued function of $t$ in $[0,1]$ which agrees with $m_{i}(t)$ at points $k / n$, where $k$ is integral, and is linear in each interval in between. By Theorem VI of [4], there exists a unique continuous matrix valued function

$$
M_{i n}(t, w)=\left(\begin{array}{ll}
A_{\text {in }}(t, w) & B_{i n}(t, w) \\
C_{i n}(t, w) & D_{i n}(t, w)
\end{array}\right)
$$

of $t$ in $[0,1]$, for each complex number $w$, such that

$$
M_{i n}(a, w) I-I=w \int_{0}^{a} M_{i n}(t, w) d m_{i n}(t)
$$

for $0 \leqq a \leqq 1$. For each fixed $a, M_{i n}(a, z)$ is a matrix valued entire function of $z$ whose entires are real for real $z$. Since $\alpha_{i n}(a)>0$ for $a>0$, $E_{i n}(a, z)=A_{i n}(a, z)-i B_{i n}(a, z)$ satisfies (1) by Lemma 9 of [4]. Similarly, there exists a unique matrix valued function $M_{i \infty}(t, w)$ such that

$$
M_{i \infty}(a, w) I-I=w \int_{0}^{a} M_{i \infty}(t, w) d m_{\imath}(t),
$$

and by the proof of Theorem VII of [4], $M_{i \infty}(a, z)=\lim M_{i n}(a, z)$ as $n \rightarrow \infty$, uniformly on bounded sets. Note that the same function $m_{i}(t)$ 
occurs in (7) as in (2) for the family $\left(E_{i}(t, z)\right)$. As in the proof of Theorem IV of [4], we must have

$$
\begin{aligned}
& B_{i}(b, z) A_{i \infty}(b, z)-A_{i}(b, z) B_{i \infty}(b, z) \\
& \quad=B_{i}(a, z) A_{i \infty}(a, z)-A_{i}(a, z) B_{i \infty}(a, x)
\end{aligned}
$$

whenever $a \leqq b$. By Theorem IX of $[4], \lim A_{i}(a, z)=1$ and $\lim B_{i}(a, z)$ $=0$ uniformly on bounded sets, as $a \searrow 0$. By the proof of Theorem I, $\lim B_{i \infty}(a, z) / A_{i \infty}(a, z)=0$ as $a \searrow 0$, uniformly on bounded sets. It follows that $B_{i}(b, z) A_{i \infty}(b, z)=A_{i}(b, z) B_{i \infty}(b, z)$ identically, and there is an entire function $G_{i}(z)$, which is real for real $z$ and has no zeros, such that $E_{i}(z)=E_{i}(1, z)=G_{i}(z) E_{i \infty}(1, z)$. Since $E_{i \infty}(1, z)=\lim E_{i n}(1, z)$, we obtain $\phi_{i}(x)=\lim \phi_{i n}(1, x)$. But the theorem has already been proved for piecewise linear functions. Therefore, $\phi_{1 n}(1, x) / x$ $\leqq \phi_{2 n}(1, x) / x$ holds for every $n$, and (5) follows on letting $n \rightarrow \infty$. This completes the proof of the theorem when each $\gamma_{i}(0)$ is finite.

In the general case, let $m_{i n}(t)$ be the matrix valued function equal to $m_{i}(t)$ for $t \geqq 1 / n(i=1,2)$ and defined by

$$
\alpha_{i n}(t)=\alpha_{i}(t), \quad \beta_{i n}(t)=\beta_{i}(1 / n), \quad \gamma_{i n}(t)=\gamma_{i}(1 / n)
$$

for $t \leqq 1 / n$. If $M_{i n}(a, z)$ is defined by $(7)$, the entire function $E_{i n}(a, z)$ $=A_{\text {in }}(a, z)-i B_{\text {in }}(a, z)$ satisfies (1) for every $n$, as we have seen. Since $\gamma_{i n}(0)$ is finite for every $n$, the inequality $\phi_{1 n}(a, x) / x \leqq \phi_{2 n}(a, x) / x$ holds by a special case of the theorem already established. The theorem will follow if we can show that $\phi_{i}(x)=\lim \phi_{i n}(1, x)$ as $n \rightarrow \infty$. To see this let $M_{i}(1 / n, t, w)$ be the unique matrix valued function of $t$ in $[1 / n, 1]$ such that

$$
M_{i}(1 / n, a, w) I-I=w \int_{1 / n}^{a} M_{i}(1 / n, t, w) d m_{i}(t)
$$

holds, for every complex number $w$. By formula (55) of [5], $M_{i n}(a, z)$ is a matrix valued entire function of $z$ such that

$$
\begin{aligned}
\left(A_{i}(a, z), B_{i}(a, z)\right) & =\left(A_{i}(1 / n, z), B_{i}(1 / n, z)\right) M_{i}(1 / n, a, z) \\
\left(A_{\text {in }}(a, z), B_{i n}(a, z)\right) & =\left(1, \alpha_{i}(1 / n) z\right) M_{i}(1 / n, a, z)
\end{aligned}
$$

holds whenever $1 / n \leqq a \leqq 1$. The desired limit $\phi_{i}(a, x)=\lim \phi_{i n}(a, x)$ will follow if we can show that

$$
B_{i}(a, z) / A_{i}(a, z)=\lim B_{i n}(a, z) / A_{\text {in }}(a, z)
$$

holds whenever $A_{i}(a, z) \neq 0$. By the Poisson representation of a function positive and harmonic in a half-plane, as used in the proof of 
Theorem I, it is sufficient to obtain (10) when $y>0$. By the proof of Lemma 9 of [4], the linear fractional transformation

$$
w \rightarrow \frac{A_{i}(1 / n, a, z)+C_{i}(1 / n, a, z) w}{B_{i}(1 / n, a, z)+D_{i}(1 / n, a, z) w}
$$

takes the half-plane $i(\bar{w}-w) \geqq 0$ onto the closed disk $\mathscr{D}_{i}(1 / n, a, z)$ of center

$$
\frac{i\left[A_{i}(1 / n, a, z) \bar{D}_{i}(1 / n, a, z)-C_{i}(1 / n, a, z) \bar{B}_{i}(1 / n, a, z)\right]}{i D_{i}(1 / n, a, z) \bar{B}_{i}(1 / n, a, z)-i B_{i}(1 / n, a, z) \bar{D}_{i}(1 / n, a, z)}
$$

and radius

$$
\frac{1}{i D_{i}(1 / n, a, z) \bar{B}_{i}(1 / n, a, z)-i B_{i}(1 / n, a, z) \bar{D}_{i}(1 / n, a, z)},
$$

and $D_{i}(1 / n, a, z)$ is contained in $D_{i}(1 / m, a, z)$ when $m \leqq n$. Because of (8) and (9), $B_{i}(a, z) / A_{i}(a, z)$ and $B_{i n}(a, z) / A_{i n}(a, z)$ both belong to $D_{i}(1 / n, a, z)$. Formula (10) follows immediately if the radius of $D_{i}(1 / n, a, z)$ goes to zero as $n \rightarrow \infty$. Otherwise, $\gamma_{i}(0)$ is finite by the proof of Theorem VIII of [4], and (10) can be obtained by arguments earlier in the proof.

\section{REFERENCES}

1. R. P. Boas, Jr., Entire functions, Academic Press, New York, 1954.

2. L. de Branges, Some Hilbert spaces of entire functions, Proc. Amer. Math. Soc. 10 (1959), 840-846.

3. - Some Hilbert spaces of entire functions, Trans. Amer. Math. Soc. 96 (1960), 259-295.

4. - Some Hilbert spaces of entire functions. II, Trans. Amer. Math. Soc. 99 (1961), 118-152.

5. - Some Hilbert spaces of entire functions. III, Trans. Amer. Math. Soc. 100 (1961), 73-115.

6. - Some Hilbert spaces of entire functions. IV, Trans. Amer. Math. Soc. 105 (1962), 43-83.

7. - Homogeneous and periodic spaces of entire functions, Duke Math. J. 29 (1962), 203-224.

8. - Symmetry in spaces of entire functions, Duke Math. J. 29 (1962), 383392.

9. - Entire functions and integral transforms, Bull. Amer. Math. Soc. 68 (1962), 103-106.

10. - Some applications of spaces of entire functions, Canad. J. Math. (to appear).

NEW YoRK UNIVERSITY 